\title{
Comparison of laser microsurgery and open partial laryngectomy for T1-2 laryngeal cancer treatment
}

\author{
Chunlin Luo, Kexing Lv, Qihong Liu, Yihui Wen, Meiya Lin, Zhangfeng Wang, Xiaolin Zhu, Aiyun Jiang, \\ Weiping Wen, Wenbin Lei
}

Department of Otolaryngology, the First Affiliated Hospital of Sun Yat-sen University, Guangzhou, China

Contributions: (I) Conception and design: C Luo, W Lei; (II) Administrative support: All authors; (III) Provision of study materials or patients: A Jiang, W Wen, W Lei; (IV) Collection and assembly of data: C Luo; (V) Data analysis and interpretation: K Lv, Q Liu, Y Wen, M Lin, Z Wang, X Zhu, A Jiang, W Wen; (VI) Manuscript writing: All authors; (VII) Final approval of manuscript: All authors.

Correspondence to: Wenbin Lei. Department of Otolaryngology, the First Affiliated Hospital of Sun Yat-sen University, No. 58, Second Zhongshan Road, Guangzhou 510080, China. Email: leiwb@mail.sysu.edu.cn.

\begin{abstract}
Background: This study aims to investigate the clinical efficacy of transoral laser microsurgery and open partial laryngectomy (OPL) in treating T1-2 laryngeal cancer.

Methods: A retrospective analysis was conducted of 182 patients with T1-2 cancer with anterior vocal commissure (AVC) involvement. The local control (LC), disease-free survival (DFS) and overall survival (OS) rates at 5-year follow-up and the influencing factors were analyzed.

Results: No significant difference was observed in the LC or DFS rates between the two groups at 3-and 5 -year follow-up. No significant difference was found between the two groups with T1-stage disease. The 5 -year LC rates were significantly different from patients with grade 3 or 4 tumors on indirect laryngoscopy and patients with class III or IV tumors on the modified Mallampati test (MMT) (log-rank test: $\chi^{2}=8.037$, $\mathrm{P}=0.005)$. The 3 -year LC rate of OPL in the depth of pathological infiltration $(3-5 \mathrm{~mm})$ group was found to be significantly higher than that of TLM. Significant differences in pathological infiltration depth $(3-5 \mathrm{~mm})$ existed between the two groups (log-rank test: $\chi^{2}=5.786, \mathrm{P}=0.016$ ).

Conclusions: T1 lesions are generally limited and superficial, and laser surgery can be well-controlled. For patients with difficult airway exposure, surgeons should have extensive surgical experience and skills. It is recommended that a variety of equipment should be ready so that surgeons can convert to open surgery at any time. For patients with a deep infiltration depth, surgeons should examine laryngoscopy imaging results before surgery.
\end{abstract}

Keywords: Laryngeal carcinoma; transoral laser microsurgery; open partial laryngectomy (OPL); anterior vocal commissure (AVC); survival rate

Submitted Dec 10, 2020. Accepted for publication Feb 26, 2021.

doi: $10.21037 / \mathrm{atm}-21-135$

View this article at: http://dx.doi.org/10.21037/atm-21-135

\section{Introduction}

Laryngeal cancer is a common malignant tumor that accounts for $4.5 \%$ of systemic malignancies, with glottic laryngeal cancer representing approximately $50 \%$ of laryngeal tumors (1). Transoral carbon dioxide laser microsurgery (TLM) is the preferred treatment for early glottic cancer (Tis) (2-4). In the past, OPL could remove tumor tissue radically, which greatly reduced the postoperative recurrence rate, but due to the loss of normal laryngeal function caused by extensive resection, the quality of life and voice of patients decreased significantly after operation. And accompanied by more postoperative complications, it takes a long time to recover. $\mathrm{CO}_{2}$ laser belongs to the infrared spectrum, which is a continuous gas molecular laser. The laser efficiency is about $10-15 \%$. 
The depth of penetration is calculated by microns, and the damage to the surrounding tissue is minimal. Due to the extremely small angle of laser emission and high energy density during the operation, the power of kilowatt per square centimeter can be obtained after focusing. This makes it have highly concentrated directional and energy characteristics, which can give full play to burning, gasification and cutting focus tissue in the treatment of early glottic carcinoma, and can seal lymphatic vessels, blood vessels and nerve endings. It can reduce the local avulsion and traction of the instrument, and maximize the normal function of the patient's larynx. The recurrence rate is relatively high when the tumor is associated with anterior vocal commissure (AVC) involvement. It is widely believed that this involvement is related to the narrow and complex structure of the AVC, in which the AVC is fused from the front of the bilateral vocal cords and attached to the anterior part of the vestibular fold. The fibers of the acoustic ligament are directly connected to the thyroid cartilage to form Broyles' ligament (5). It has been reported that the former condition, combined with Broyles' ligament, disrupts the integrity of the thyroid cartilage membrane, leading to AVC susceptibility $(6,7)$. Other researchers have suggested that this phenomenon has a 'barrier effect' (8). Studies have shown that the thyroepiglottic ligament in the superior glottic area is similar to Broyles' ligament in the glottic area, but there is no continuity between glands and vessels (9). Wu et al. performed a pathological biopsy to verify the latter perspective and found that the ligament had a certain 'barrier effect' on tumor cells; however, this effect was weakened if the vocal cord muscles were disrupted (10). Therefore, it is important to determine the efficacy of TLM in early-stage laryngeal carcinoma with AVC involvement. In this research, we analyzed clinical data from the past 10 years to evaluate the clinical efficacy of TLM in patients with anterior vocal commissure (AVC) cancer. The results were compared with those obtained for patients who underwent open partial laryngectomy (OPL) to select an optimal treatment method for patients with AVC involvement.

We present the following article in accordance with the STROBE reporting checklist (available at http://dx.doi. org/10.21037/atm-21-135).

\section{Methods}

\section{General information}

A total of 182 patients were diagnosed with AVC involvement in early glottic carcinoma at our unit from January 2006 and January 2017. Patients were divided into TLM and OPL groups, with 65 and 117 cases in each group, respectively. The TLM group was implemented in 2010, while OPL group was implemented in 2006. The baseline characteristics of patients in the OPL group between 2006 and 2010 were no different from those in the same group after 2010. Regarding TNM stage (AJCC/ UICC) classification, 63 cases had T1a, 72 cases had T1b, and 47 cases had T2. The related data are listed in Table 1 .

\section{Conditions for AVC assessment}

AVC involvement was diagnosed with fiberoptic laryngoscopy, CT and/or MRI, and/or pre-associated pathology, or postoperative pathology.

\section{Exclusion criteria}

(I) Non-squamous cell carcinoma; (II) clinical stages not T1-2N0M0 (according to the 2002 AJCC/UICC); (III) patients with oropharyngeal surgery; (IV) patients with severe abnormalities in vital organs, such as heart, brain and lung; and (V) patients unwilling to participate in the study.

\section{Measurement of the tumor infiltration depth of pathological specimens}

According to the specimen locations, the infiltrated tissues of the surgically resected tumor specimens were taken from tissues that were vertically infiltrated. The sections were cut, fixed, and preserved, before being dehydrated, paraffinembedded, and cut into 3- $\mu \mathrm{m}$ thick slices. Hematoxylin and eosin staining was performed. The depth of invasion was measured as the distance from the epithelial location to the deepest tumor tissue, and the data were recorded.

\section{Surgical methods}

Zeiss S88 surgical microscope (Zeiss, Germany), Sharplan$30 \mathrm{c} \mathrm{CO}_{2}$ laser and coupler (Israel Scientific), support laryngoscope (STORZ, Germany), laryngoscope for microsurgery. The methods were in accordance with the 2007 European Laryngeal Society classification criteria for $\mathrm{CO}_{2}$ laser vocal cord resection. These procedures include frontal laryngectomy (FLPL), partial cartilage upper laryngectomy, modified ring epiglottic bone fixation (SCPLCTP), and a modified thyroid cartilage window (WPL) 
Table 1 Distribution of patient characteristics

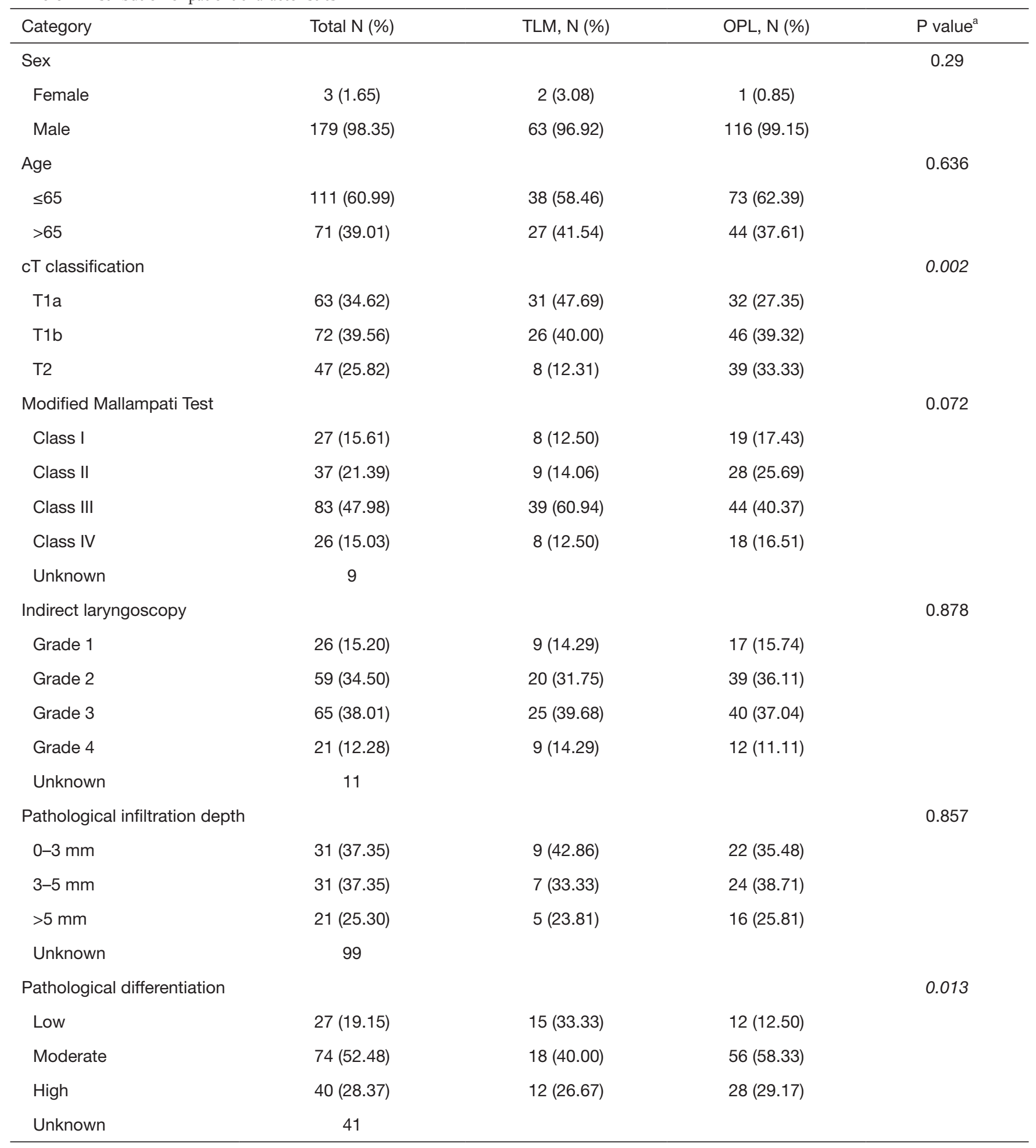

${ }^{a}$, italic indicates $\mathrm{P}<0.05$. The differences between the two groups in each category were statistically significant by Fisher's exact test. TLM, transoral carbon dioxide laser microsurgery; OPL, open partial laryngectomy. 
(11-15). A total of 65 cases underwent TLM. The OPL group included 48 cases of modified WPL, 37 cases of CTP, and 32 cases of FLPL. All surgeries were performed by one experienced surgeon using indistinguishable technique.

\section{Research process}

Relevant data and an OPL case-control study were analyzed to study the surgical efficacy of TLM in early glottic laryngeal cancer with AVC involvement. All patients signed an informed consent form. Twelve months (or more) after the initial surgical treatment, an outpatient visit or telephone call was arranged to verify survival for comparison analysis between groups. In addition, outpatient follow-up assessments were performed, including the modified Mallampati test (MMT) and indirect laryngoscopy (IL), with all data collected and recorded. Patients or their family members filled in the quality of life scales QLQ-C30 (V3.0) and QLQ-H\&N35 (V1.0). All procedures performed in this study involving human participants were in accordance with the Declaration of Helsinki (as revised in 2013). The study was approved by ethics board of The First Affiliated Hospital of Sun Yat-sen University (No.: ChiCTR-ORC-17010515).

\section{MMT method}

Patients sat opposite the observer and outstretched their tongues to maximal extension (not pronounced). The classification was based on the structures observed: class I, which included soft palate, fauces, uvula, and tonsillar pillars; class II, which included soft palate, fauces and base of the uvula; class III, which included soft palate, base of the uvula; and class IV, whereby the soft palate was not visible.

\section{Indirect laryngoscopy (IL)}

The airway view as shown by IL was classified as using the following grades: grade 1, full view of the glottis (vocal cords visible); grade 2, posterior commissure visible; grade 3, epiglottis visible; grade 4, no glottic structure visible.

\section{QLQ-C30 (V3.0) Scale}

This questionnaire consisted of 30 items: five on functional scales, including physical function (PF), role function (RF), cognitive function (CF), emotional function (EF), and social function (SF); three on symptom scales, including fatigue (FA), pain (PA), nausea, and vomiting (NV); six on individual items, including dyspnea (DY), insomnia (SL), loss of appetite (AL), constipation (CO), diarrhoea (DI), and financial difficulties (FD); and one overall rate, which is the quality of life scale (GQL).

\section{QLQ-H\&N35 (V1.0) Scale}

This scale consisted of 35 items. These were divided into 18 fields: 7 symptom areas, including pain (HNPA), swallowing (HNSW), sense problems (HNSE), speech problems (HNSP), trouble with social eating (HNSO), trouble with social contact (HNSC), and less sexuality (HNSX); and 11 separate items, including teeth (HNTE), opening mouth (HNOM), dry mouth (HNDR), sticky saliva (HNSS), coughing (HNCO), felt ill (HNFI), painkillers (HNPK), nutritional supplements (HNNU), feeding tubes (HNFE), weight loss (HNWL), and weight gain (HNWG).

\section{Evaluated parameters}

Local control (LC), disease-free survival (DFS), and overall survival (OS) rates were calculated.

\section{Statistical analysis}

All data were analyzed using R 3.4.3 (R Core Team, Vienna, Austria). The Kaplan-Meier method was used to estimate LC, DFS, OS rates and survival rates at specific time points. The log-rank test was used to compare survival rates between groups, with $\mathrm{P}<0.05$ indicating statistical significance. The rank-sum test was used for quality of life comparisons between groups, with $\mathrm{P}<0.05$ indicating statistical significance.

\section{Results}

\section{Comparison of LC, DFS and OS rates between groups}

As shown in Table 2, among the 182 patients, LC rates at 1, 3 , and 5 years postoperatively were $96.92 \%, 85.02 \%$, and $85.02 \%$ for TLM group and $98.29 \%, 95.54 \%$, and $93.15 \%$ for OPL group, respectively. DFS rates at 1-, 3-, and 5-year postoperatively were $96.92 \%, 85.02 \%$, and $85.02 \%$ for TLM group and $98.29 \%, 92.71 \%$, and $90.39 \%$ for OPL group, respectively. There was no significant difference in OS rates between the two groups (Table 2). 
Table 2 Comparison of the survival rate between the two groups

\begin{tabular}{|c|c|c|c|}
\hline Time & OS $(95 \% \mathrm{Cl})$ & DFS (95\% Cl) & LC $(95 \%$ Cl) \\
\hline \multicolumn{4}{|l|}{ TLM } \\
\hline 1 year & $100(100,100)$ & $96.92(92.81,100)$ & $96.92(92.81,100)$ \\
\hline 3 years & $100(100,100)$ & $85.02(75.76,95.4)$ & $85.02(75.76,95.4)$ \\
\hline 5 years & $94.74(85.21,100)$ & $85.02(75.76,95.4)$ & $85.02(75.76,95.4)$ \\
\hline \multicolumn{4}{|l|}{ OPL } \\
\hline 1 year & $100(100,100)$ & $98.29(95.97,100)$ & $98.29(95.97,100)$ \\
\hline 3 years & $99.02(97.15,100)$ & $92.71(87.96,97.72)$ & $95.54(91.79,99.45)$ \\
\hline 5 years & $96.49(92.66,100)$ & $90.39(84.84,96.3)$ & $93.15(88.3,98.26)$ \\
\hline \multicolumn{4}{|l|}{$P$ value ${ }^{a}$} \\
\hline 5 years & 0.375 & 0.176 & 0.073 \\
\hline
\end{tabular}

${ }^{a}$, the LC, DFS and OS rates and the survival rates at specific time points were analyzed using the $Z$ test via the Kaplan-Meier method. TLM, transoral carbon dioxide laser microsurgery; OPL, open partial laryngectomy; LC, local control rate; DFS, disease-free survival rate; OS, overall survival rate; $\mathrm{Cl}$, confidence interval.

Table 3 Local control rate in the two groups according to different factors (\%)

\begin{tabular}{|c|c|c|c|c|c|c|c|c|c|c|c|c|}
\hline Time & \multicolumn{2}{|c|}{ Age } & \multicolumn{3}{|c|}{ cT classification } & \multicolumn{4}{|c|}{ Difficult airway } & \multicolumn{3}{|c|}{ Pathological infiltration depth } \\
\hline \multicolumn{13}{|l|}{ TLM } \\
\hline 1 year & 100 & 92.59 & 96.77 & 96.15 & 100 & 96.77 & 100 & 100 & 100 & 100 & 85.71 & 80 \\
\hline 3 years & 83.01 & 87.15 & 89.84 & 80.11 & 85.71 & 75.47 & 100 & 100 & 92.31 & 100 & 51.43 & 60 \\
\hline \multicolumn{13}{|l|}{ OPL } \\
\hline 1 year & 98.63 & 97.73 & 96.88 & 97.83 & 100 & 100 & 100 & 90 & 100 & 95.45 & 100 & 100 \\
\hline 3 years & 95.49 & 95.45 & 96.88 & 93.21 & 97.22 & 97.14 & 100 & 90 & 100 & 95.45 & 90.51 & 100 \\
\hline 5 years & 90.94 & 95.45 & 96.88 & 90.39 & 93.33 & 97.14 & 91.67 & 90 & 100 & 95.45 & 90.51 & 100 \\
\hline
\end{tabular}

Da1 grades 3 and 4 were determined via indirect laryngoscopy, and classes III and IV were determined via the modified Mallampati test; Da2 grades 1 and 2 were determined via indirect laryngoscopy, and classes III and IV were determined via the modified Mallampati test; Da3 grades 3 and 4 were determined via indirect laryngoscopy, and classes I and II were determined via the modified Mallampati test; and Da4 grades 1 and 2 were determined via indirect laryngoscopy, and classes I and II were determined via the modified Mallampati test. a, follow-up time of the study subjects did not reach the corresponding time. TLM, transoral carbon dioxide laser microsurgery; OPL, open partial laryngectomy. MMT, Modified Mallampati Test; IL, indirect laryngoscopy.

\section{Comparison of the 3-and 5-year LC rates between groups}

As shown in Table 3, no significant difference was observed between the two groups with respect to T1a-stage disease (log-rank test: $\chi^{2}=1.144, \mathrm{P}=0.285$ ). The 5 -year $\mathrm{LC}$ rates after surgery were $96.9 \%$ and $89.8 \%$ in OPL and TLM groups, respectively. Subsequent analysis showed no significant difference between the two groups with respect to T1bstage disease (log-rank test: $\left.\chi^{2}=0.864, \mathrm{P}=0.353\right)$. The LC rates at 5 years postoperatively were $90.4 \%$ and $80.1 \%$ in 


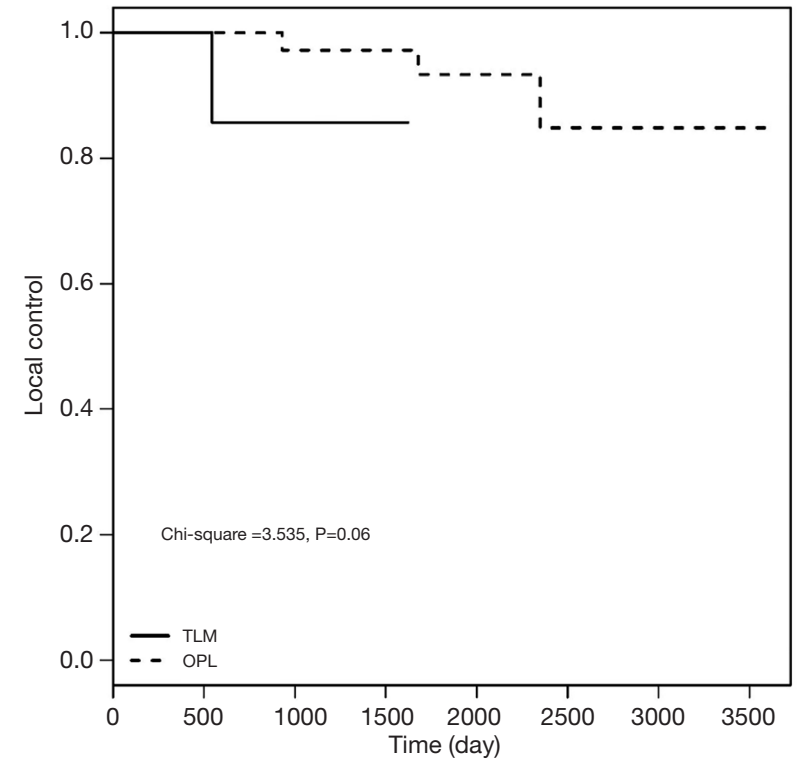

Figure 1 Comparison of the LC rates of T2-stage patients between the two groups. LC rates in the transoral carbon dioxide laser microsurgery (TLM) group (solid line) and open partial laryngectomy (OPL) group (dotted line); Kaplan-Meier analysis of the local control rates of T2 lesions in the two groups was performed (log-rank test, $\mathrm{P}=0.06)$. LC, local control.

OPL and TLM groups, respectively. The LC rates for T2stage patients for the two groups at 3 years postoperatively were $97.2 \%$ and $85.71 \%$, respectively, and the difference between the two groups was not statistically significant (logrank test: $\chi^{2}=3.535, \mathrm{P}=0.06$ ). Among patients with IL grades 3 and 4 , or MMT classes III and IV, the LC rates at 5 years postoperatively were $97.1 \%$ and $75.5 \%$ in OPL and TLM groups, respectively, with a statistically significant difference between the two groups (log-rank test: $\chi^{2}=8.037, \mathrm{P}=0.005$ ). The LC rates at 3 years postoperatively for the group with a pathological invasion depth of $3-5 \mathrm{~mm}$ were $90.5 \%$ and $51.4 \%$ for OPL and TLM, respectively, with a statistically significant difference between the two groups (log-rank test: $\left.\chi^{2}=5.786, \mathrm{P}=0.016\right)$ (Figures $\left.1-3\right)$.

\section{Comparison of the 3-and 5-year DFS rates between the two groups}

As shown in Table 4 and Figure 4, the DFS results were consistent with the $\mathrm{LC}$ rate results.

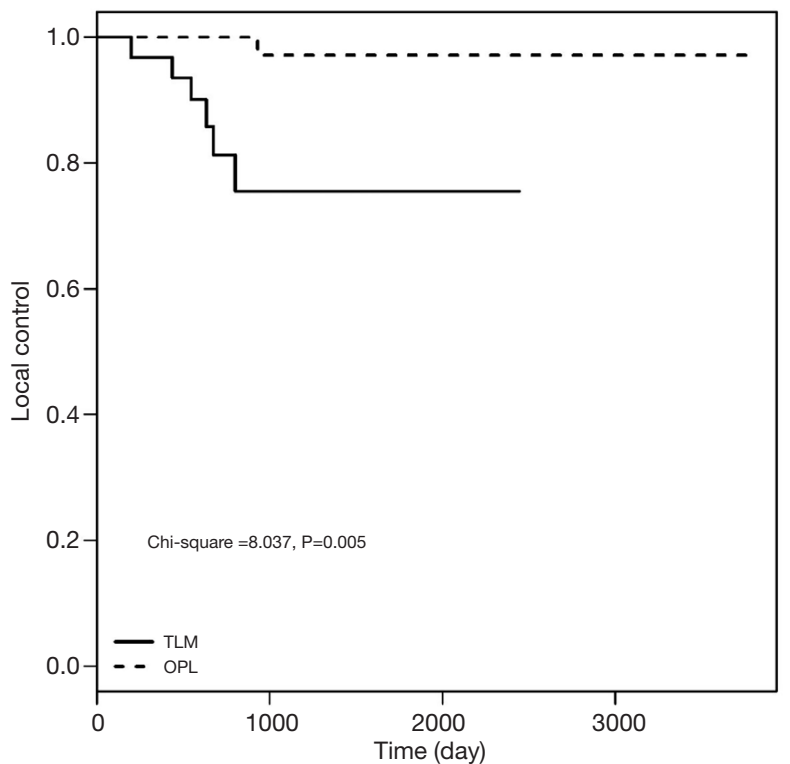

Figure 2 Comparison of LC rates of patients classified as grades 3 and 4 on indirect laryngoscopy or classes III and IV on the modified Mallampati test between the two groups. LC rates in the transoral carbon dioxide laser microsurgery (TLM) group (solid line) and open partial laryngectomy (OPL) group (dotted line); Kaplan-Meier analysis of the local control rates in patients in the two groups classified as grades 3 and 4 on indirect laryngoscopy or classes III and IV on the modified Mallampati test was performed (log-rank test, $\mathrm{P}=0.005$ ). LC, local control.

\section{Follow-up outcomes}

During early glottic carcinoma with AVC involvement in TLM patients, eight cases showed recurrence, of which three underwent OPL and five underwent total laryngectomy. One of the eight patients died due to lung metastasis. One patient also had colorectal cancer, and one patient failed to attend for follow-up. In the OPL group, recurrence and metastasis occurred in nine patients, of which one patient received gamma knife treatment, four patients underwent total laryngectomy, two patients metastasized, and two patients underwent radiochemotherapy. Two patients died, with one due to carotid artery rupture and the other due to lung metastasis. Four patients developed lymph node, lung, and liver metastases. Four patients failed to attend for follow-up. In addition, only 138 patients were included health-related 


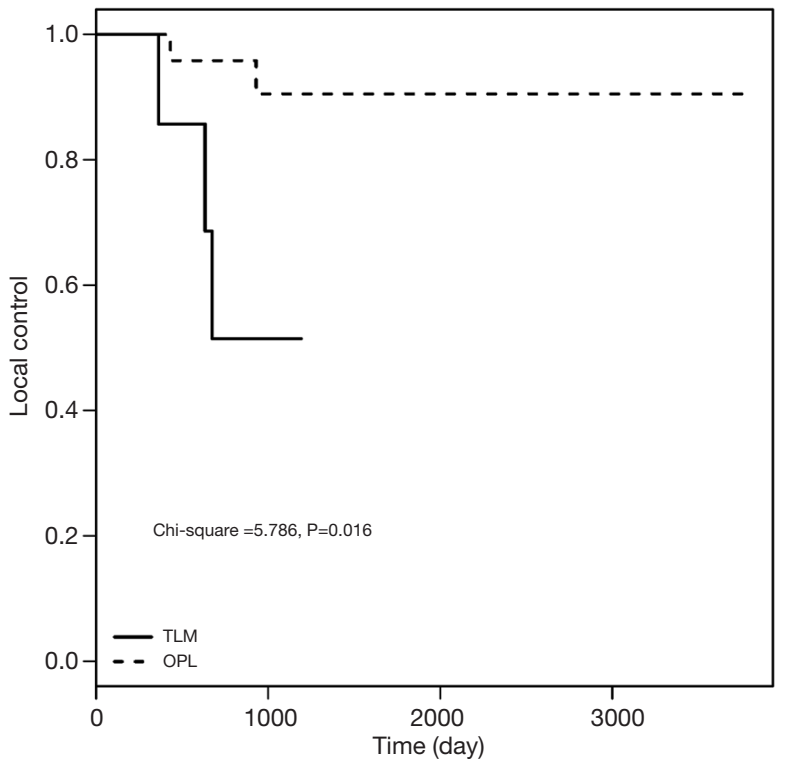

Figure 3 Comparison of LC rates of patients with pathological infiltration depths of 3-5 $\mathrm{mm}$ between the two groups. Local control rates in the transoral carbon dioxide laser microsurgery (TLM) group (solid line) and open partial laryngectomy (OPL) group (dotted line); Kaplan-Meier analysis of the local control rates in patients in the two groups with pathological infiltration depths of 3-5 mm was performed (log-rank test, $\mathrm{P}=0.016$ ). LC, local control. quality of life (HRQOL) measurement to reduce bias, exclusions made for tumor recurrence, metastasis and death. In TLM, eight patients relapsed, metastasized or died and nine patients were unable to be contacted at followup. In OPL, recurrence and metastasis led to the death of 11 patients, and 16 patients were lost during follow-up. Finally, 48 patients were included in the TLM group and 90 patients were included in OPL group. Regarding patient quality of life, the overall health quality was higher in TLM group than in OPL group (Table 5).

\section{Discussion}

Since Strong and Jako commenced TLM for glottic laryngeal cancer treatment in the 1970s (16), the use of OPL has been declining (13). Laser surgery accounts for approximately $30-50 \%$ of all laryngeal cancer treatments in developed countries. A large number of reports showed that the $\mathrm{LC}$ rate of $\mathrm{T} 1$ patients is $77-92 \%$, the $\mathrm{LC}$ rate of T2 patients is $66-88 \%$, and larynx preservation rate is $90-99 \%(17-21)$. In addition, the LC rate of OPL is $86-98 \%$, and the LFP rate is $88-100 \%(12,22-25)$. Despite its success, the efficacy of TLM in the treatment of this type of carcinoma with AVC involvement remains unclear. Most scholars believe that AVC glottic cancer requires open

Table 4 Disease-free survival rates in the two groups according to different factors (\%)

\begin{tabular}{|c|c|c|c|c|c|c|c|c|c|c|c|c|}
\hline Time & \multicolumn{2}{|c|}{ Age } & \multicolumn{3}{|c|}{ cT classification } & \multicolumn{4}{|c|}{ Difficult airway } & \multicolumn{3}{|c|}{ Pathological infiltration depth } \\
\hline \multicolumn{13}{|l|}{ TLM } \\
\hline 1 year & 100 & 92.59 & 96.77 & 96.15 & 100 & 96.77 & 100 & 100 & 100 & 100 & 85.71 & 80 \\
\hline 3 years & 83.01 & 87.15 & 89.84 & 80.11 & 85.71 & 75.47 & 100 & 100 & 92.31 & 100 & 51.43 & 60 \\
\hline \multicolumn{13}{|l|}{ OPL } \\
\hline 1 year & 98.63 & 97.73 & 96.88 & 97.83 & 100 & 100 & 100 & 90 & 100 & 95.45 & 100 & 100 \\
\hline 3 years & 92.3 & 93.13 & 90.3 & 90.55 & 97.22 & 97.14 & 100 & 90 & 100 & 90.43 & 90.51 & 100 \\
\hline 5 years & 87.91 & 93.13 & 90.3 & 87.81 & 93.33 & 97.14 & 91.67 & 90 & 100 & 90.43 & 90.51 & 100 \\
\hline
\end{tabular}

Da1 grades 3 and 4 were determined via indirect laryngoscopy, and classes III and IV were determined via the modified Mallampati test; Da2 grades 1 and 2 were determined via indirect laryngoscopy, and classes III and IV were determined via the modified Mallampati test; Da3 grades 3 and 4 were determined via indirect laryngoscopy, and classes I and II were determined via the modified Mallampati test; and Da4 grades 1 and 2 were determined via indirect laryngoscopy, and classes I and II were determined via the modified Mallampati test. ", follow-up time of the study subjects did not reach the corresponding time. TLM, transoral carbon dioxide laser microsurgery; OPL, open partial laryngectomy; MMT, Modified Mallampati Test; IL, indirect laryngoscopy. 


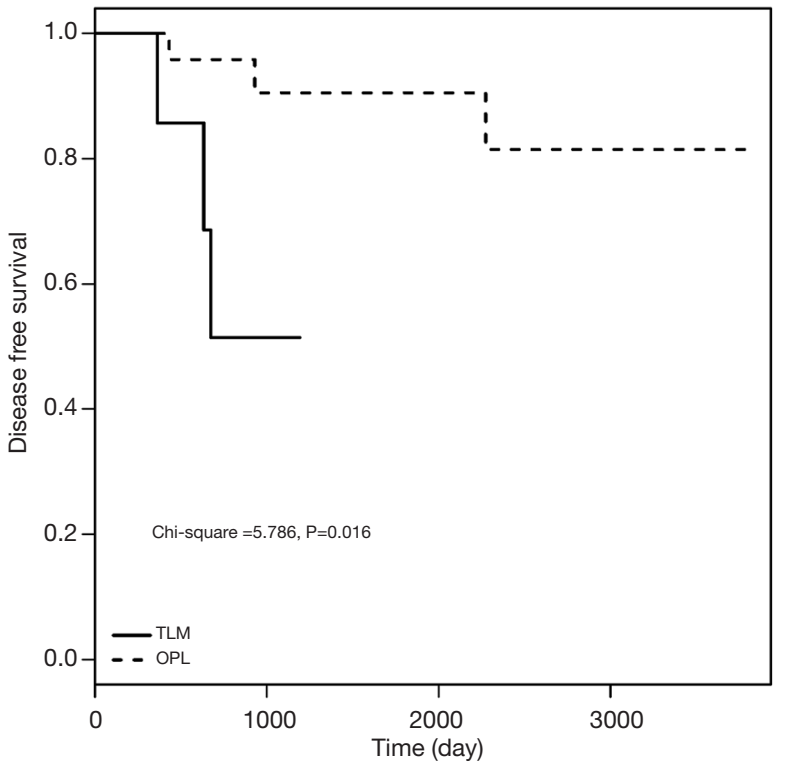

Figure 4 Comparison of the DFS rates of patients with pathological infiltration depths of $3-5 \mathrm{~mm}$ between the two groups. Disease-free survival rates in the transoral carbon dioxide laser microsurgery (TLM) group (solid line) and open partial laryngectomy (OPL) group (dotted line); Kaplan-Meier analysis of the disease-free survival rates in patients in the two groups with pathological infiltration depths of 3-5 mm was performed (log-rank test, $\mathrm{P}=0.016)$. DFS, disease-free survival.

surgery or radiotherapy and that TLM can only be used for AVC involvement in laryngeal cancer; however, other scholars believe that TLM can be appropriate if certain conditions, such as AVC exposure, are met. The purpose of this retrospective study was to investigate the effect of TLM on treating early-stage glottic carcinoma with AVC involvement.

\section{AVC involvement and TNM staging}

The UICC or AJCC staging of laryngeal cancer is widely used; however, the application of TNM staging in cases with AVC involvement remains controversial. Some scholars believe that TNM staging has no significant difference in $\mathrm{LC}$ and survival rates and can therefore be performed in accordance with the original stage $(2,19,26-28)$; however, other scholars believe that AVC involvement may also be associated with a high recurrence rate (6). Karatzanis et al. reported that TNM staging did not sufficiently encompass AVC lesions and that TNM staging systems including
AVC lesions should be developed (4). This study is based on TNM staging and AVC involvement. As shown in Table 2, no significant difference was found between TLM and OPL groups on OS, DFS and LC rates. As shown in Table 3, the 5-year LC of T1b was the lowest in the TLM group, which was related to the extent of preoperative resection or insufficiency of prediction. In general, for T1 disease, no statistically significant difference was observed between the TLM group and OPL groups. Therefore, T1 lesions are generally considered limited and superficial, and laser treatment can be well-controlled. In this analysis, Tis cases were excluded because few Tis patients choose open surgery, making the comparison difficult. The LC rate of T2 patients in the TLM group was $85.71 \%$, consistent with previous data from Steiner, and was significantly different from the LC rate of T2 patients in the OPL group (29). Since the laser group included only eight $\mathrm{T} 2$ cases, the difference was not significant and additional data analysis is needed with a larger sample size. In addition, one case of recurrence was observed among the eight $\mathrm{T} 2$ cases, and this case was classified as grade 4 by IL and MMT. Therefore, the difficulty of exposure might have been the cause of $\mathrm{T} 2$ recurrence. We believe that $\mathrm{CO}_{2}$ laser application for $\mathrm{T} 2$ cases is conditional. Firstly, TLM was fully evaluated before surgery and based on well-predicted difficult exposure, and the AVC lesion could be fully exposed during surgery. Secondly, preoperative laryngeal CT or MRI was performed to assess the extent of the tumor and to ensure that the surgical margin was more than $3 \mathrm{~mm}$ to achieve en bloc tumor resection. Thus, under certain conditions, including a surgeon with extensive clinical experience and high skill level, TLM can achieve better results.

\section{Difficult exposure}

The application of TLM, especially with inadequate preoperative or intraoperative assessment of the AVC range, can lead to recurrence. Sachse et al. found that in patients with early laryngeal cancer, the difference of LC rates between laser and open surgery was mainly due to poor glottic exposure (30).

Various evaluation methods are available to determine whether an airway is difficult, such as the Cormack-Lehane system and thyromental distance. The specificity and sensitivity of the MMT are reportedly low, thus limiting its clinical application (31). Therefore, the MMT needs to be combined with other methods to better predict difficult airways (32). Yamamoto et al. compared MMT score, IL and 
Table 5 Correlation with the life quality in the two groups

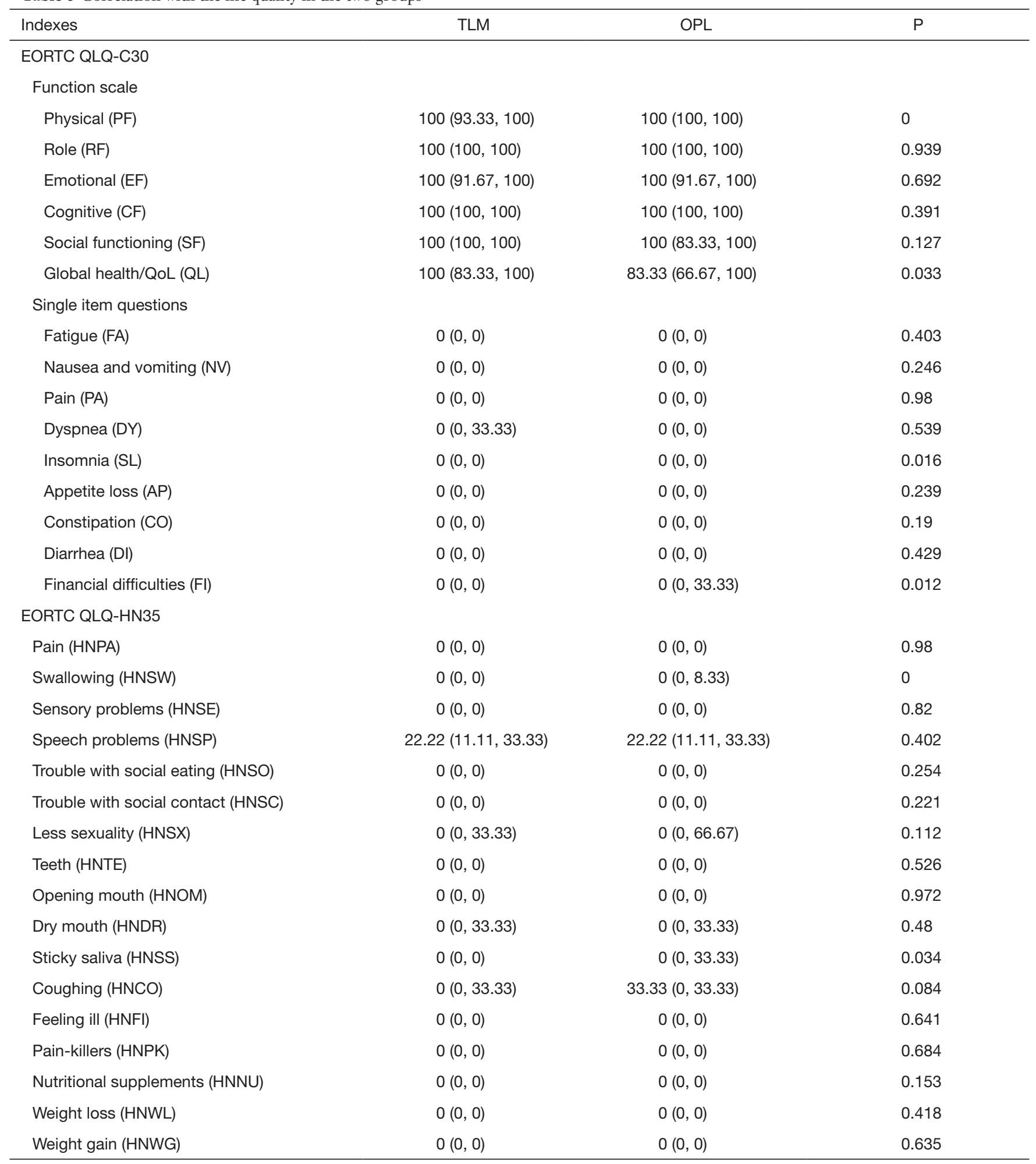

TLM group was better than the OPL group in PI, QOL, SL, FI, HNSW and HNSS, which were significantly different. The rank-sum test was used to compare the groups, and $\mathrm{P}<0.05$ means statistically significant. TLM, transoral carbon dioxide laser microsurgery; OPL, open partial laryngectomy. 
Wilson risk sum score to predict difficult airways, and the IL method was found to have the highest reliability (33). The present study examined the prognosis of patients who were treated by different methods and who were classified as grade 3 or 4 according to the MMT and IL. The LC rate at 5 years postoperatively was significantly different between the two groups. Therefore, grade 3 or 4 was considered an indicator of difficult airway exposure. In addition, the LC rate at 5 years postoperatively was $76.8 \%$ for patients in the TLM group who were classified as grade 3 or 4 by IL alone, which is similar to the result of the combined grade 3 and 4 groups. In fact, there is no single evaluation method that can accurately predict difficult airway before surgery. Moreover, it is necessary to consider other influencing factors, such as the depth of anaesthesia and dosage. In general, for patients with difficult airway exposure, surgeons should have extensive surgical experience and skill. It is highly recommended that a variety of laryngoscopes and equipment are ready to allow the surgeon to convert to open surgery at any time if required.

\section{Surgical margin}

TLM has gradually become one of the main ways to treat early glottic carcinoma because of its many advantages, such as less trauma, overall postoperative condition, less hospitalization cost, less intraoperative and postoperative complications and so on. However, it has been difficult to have a consensus on the invasion of the anterior union and some patients with stage T2. According to the theoretical analysis, the tumor tissue exposed by bracing laryngoscope can be removed by laser during the operation, but it is found in the process of clinical treatment for this type of patients. Due to the difficulty of intraoperative exposure and the relative limitations of TLM, it is difficult to achieve ideal resection, resulting in a relatively high recurrence rate. There is wide debate regarding the impact of the surgical margin on patient prognosis. Fiz et al. first proposed the definition of positive margins in tissue specimens and noted that the depth of tumor infiltration is correlated to patient prognosis $(34,35)$. He reported that the positive margin rate of pathological tissue specimens obtained from lung cancer $\mathrm{CO}_{2}$ laser ablation reached $50 \%$. It should be noted that the pathological margin is different from the safe surgical margin; the clinical significance of the former is still controversial and requires further investigation, while the positive outcomes of the latter have been established. Grant et al. reported that samples could be interpreted as positive by pathologists, potentially misleading surgeon and patient judgment concerning prognosis and the need for adjuvant therapy (36). Therefore, for patients with early glottic laryngeal laser surgery, a report of a positive tumor margin does not necessarily indicate incomplete tumor resection (34), while a negative result does not necessarily guarantee complete resection. In this study, we analyzed the margins of the two groups and observed positive, suspiciouspositive and negative margins according to the standard. We found that the margins had no direct correlation with LC and DFS rates. Therefore, for patients with suspiciouspositive margins, close follow-up is required after surgery. Due to the small sample size $(n=3)$ of positive cases in this study, no statistical significance can be concluded and a larger sample size is needed for future research.

\section{Pathological infiltration depth}

Steiner et al. reported inconsistent prognosis between patients presenting with the same stage of cancer (27). This discrepancy is related to the size and shape of the tumor and the invasion depth. Regarding the infiltration depth of glottic laryngeal carcinoma, no previous data analysis has been performed, and no investigative analysis of survival rates is available. In this study, submucosal infiltration was analyzed, and the 3-year LC rate of OPL in the depth of pathological infiltration $(3-5 \mathrm{~mm}$ ) group was found to be significantly higher than that of TLM. Therefore, for patients with pathological invasion depth greater than $3 \mathrm{~mm}$, the risk of residual tumor recurrence increases. There are several explanations for this, including a lack of preoperative CT or MRI evaluation by surgeons, poorly defined boundaries between the tissue and the tumor, or lack of postoperative specimen to determine the completeness of tumor resection. This retrospective study examined the infiltration depth, and complete specimens were obtained from 83 patients, including 62 patients in the OPL group and 21 patients in the TLM group. Patients who underwent TLM participated in follow-up. Seven patients with a pathological infiltration depth of 3-5 mm were reported, and three of them had recurrence. Among them, one patient with total laryngectomy had recurrence 21 months postoperatively, one patient with partial laryngectomy had recurrence 23 months postoperatively, and all patients survived. The latter patient underwent total laryngectomy 12 months postoperatively and died of a pulmonary metastasis 48 months later. There were five patients with pathological infiltration depth of $5 \mathrm{~mm}$ or 
more and two of them had recurrence, of which one patient underwent total laryngectomy 7 months after the operation and survived, while the other patient underwent partial laryngectomy 15 months after recurrence and did not attend follow-up. Therefore, for tumors with deeper infiltration depth, it is necessary to increase the frequency of follow-up to monitor recurrence. If recurrence or metastasis is found, patients should consider further surgical, radiotherapy, chemotherapy or combination therapy options.

\section{Age, sex, and lymph node dissection as tumor differentiation factors}

No significant difference was found in LC and survival rates between different age groups. No significant difference was found in LC and DFS rates regardless of whether lymph node dissection was or was not performed. The mechanism underlying these findings for most lesions remains unknown because T3 and T4 cases were not included in this study. The analysis of tumor pathological differentiation showed that no significant difference in DFS and LC rates existed among well-differentiated, moderately differentiated, and poorly differentiated tumors, which might relate to the partial absence of case data.

\section{Health-related quality of life (HRQOL)}

In addition to prolonged survival time, quality of life is also an important consideration for patients with head and neck cancer. For early glottic laryngeal carcinoma with AVC involvement, little research has compared quality of life between TLM and OPL groups. Usually EORTC QLQ-C30 questionnaire and the QLQ-H\&N35 questionnaire are used to evaluate quality of life for patients. Those questionnaires have also been translated into Chinese, with good reliability and validity in clinical practice $(37,38)$. Regarding HRQOL after laryngeal cancer surgery, Luo et al. showed that typical side effects of postoperative laryngeal cancer included pain, dry mouth and speech disorder (39). Lundström et al. reported that global health and quality of life was associated with dry mouth, difficulty swallowing, and trouble with social contact (40). Bindewald et al. summarized the finding from 371 patients who underwent open surgery and who experienced notable side effects from postoperative radiotherapy complications, such as sense problems, dry mouth, sticky saliva, and oropharyngeal pain. Additionally, factors that contributed to HRQOL were related to the tumor staging and whether patients took postoperative radiotherapy or tracheostomy (41). This study was focused on early glottic carcinomas with AVC involvement. A total of 138 patients were not treated with postoperative radiotherapy. The patients were successfully extubated after open surgery. The results showed that HRQOL was significantly better for TLM than for OPL in the QLQ-HN35, while in the QLQHN35, TLM was superior in dysphagia and sticky saliva compared with OPL. Both differences were statistically significant. Therefore, patients who have open surgery may experience difficulty in swallowing and lower quality of life, and should be closely monitored after surgery. HRQOL of patients who had TLM is better than that of patients who have OPL; however, if there is difficulty in exposing the laryngoscope and deep infiltration, it is still necessary to choose laser surgery carefully.

\section{Research limitations}

Several limitations exist in this study. Firstly, in the analysis of related factors, some data were not available which might have led to false-positive results. Thirdly, some pathologists did not measure the depth, resulting in incomplete data; further specification of sampling and observation time is also required due to a lack of universal standard. Finally, further follow-up is required to compare the long-term efficacy between the two groups.

\section{Conclusions}

T1 lesions are generally limited and superficial, and laser surgery can be well-controlled. For patients with difficult airway exposure, surgeons should have extensive surgical experience and skills. It is recommended that a variety of equipment should be ready so that surgeons can convert to open surgery at any time. For patients with a deep infiltration depth, surgeons should examine laryngoscopy imaging results before surgery.

\section{Acknowledgments}

Funding: This work was supported by grants from the 5010 Clinical Research Program of Sun Yat-sen University (2017004), the Natural Science Foundation of Guangdong Province of China (2016A030313247), the Science and Technology Program of Guangzhou of 
China (201704020092) and the Science and Technology Planning Project of Guangdong Province of China (2017A050506018).

\section{Footnote}

Reporting Checklist: The authors have completed the STROBE reporting checklist. Available at http://dx.doi. org/10.21037/atm-21-135

Data Sharing Statement: Available at http://dx.doi. org/10.21037/atm-21-135

Conflicts of Interest: All authors have completed the ICMJE uniform disclosure form (available at http://dx.doi. org/10.21037/atm-21-135). The authors have no conflicts of interest to declare.

Ethical Statement: The authors are accountable for all aspects of the work in ensuring that questions related to the accuracy or integrity of any part of the work are appropriately investigated and resolved. All procedures performed in this study involving human participants were in accordance with the Declaration of Helsinki (as revised in 2013). The study was approved by ethics board of The First Affiliated Hospital of Sun Yat-sen University (No.: ChiCTR-ORC-17010515). All patients signed an informed consent form.

Open Access Statement: This is an Open Access article distributed in accordance with the Creative Commons Attribution-NonCommercial-NoDerivs 4.0 International License (CC BY-NC-ND 4.0), which permits the noncommercial replication and distribution of the article with the strict proviso that no changes or edits are made and the original work is properly cited (including links to both the formal publication through the relevant DOI and the license). See: https://creativecommons.org/licenses/by-ncnd/4.0/.

\section{References}

1. Schultz P. Vocal fold cancer. Eur Ann Otorhinolaryngol Head Neck Dis 2011;128:301-8.

2. Galli A, Giordano L, Sarandria D, et al. Oncological and complication assessment of $\mathrm{CO} 2$ laser-assisted endoscopic surgery for T1-T2 glottic tumours: clinical experience. Acta Otorhinolaryngol Ital 2016;36:167-73.
3. Hartl DM, Brasnu DF. Contemporary surgical management of early glottic cancer. Otolaryngol Clin North Am 2015;48:611-25.

4. Karatzanis AD, Psychogios G, Zenk J, et al. Comparison among different available surgical approaches in T1 glottic cancer. Laryngoscope 2009;119:1704-8.

5. Rucci L, Gammarota L, Borghi Cirri MB. Carcinoma of the anterior commissure of the larynx: I. Embryological and anatomic considerations. Ann Otol Rhinol Laryngol 1996;105:303-8.

6. Andrea M, Guerrier Y. The anterior commissure of the larynx. Clin Otolaryngol Allied Sci 1981;6:259-64.

7. Krespi YP, Meltzer CJ. Laser surgery for vocal cord carcinoma involving the anterior commissure. Ann Otol Rhinol Laryngol 1989;98:105-9.

8. Kirchner JA, Carter D. Intralaryngeal barriers to the spread of cancer. Acta Otolaryngol 1987;103:503-13.

9. Andrea M. Vasculature of the anterior commissure. Ann Otol Rhinol Laryngol 1981;90:18-20.

10. Wu J, Zhao J, Wang Z, et al. Study of the histopathologic characteristics and surface morphologies of glottic carcinomas with anterior vocal commissure involvement. Medicine (Baltimore) J 2015;94:e1169.

11. Giovanni A, Guelfucci B, Gras R, et al. Partial frontolateral laryngectomy with epiglottic reconstruction for management of early-stage glottic carcinoma. Laryngoscope 2001;111:663-8.

12. Rebeiz EE, Wang Z, Mcgilligan JA, et al. Preliminary clinical results of window partial laryngectomy: a combined endoscopic and open technique. Ann Otol Rhinol Laryngol 2000;109:123-7.

13. Silver CE, Beitler JJ, Shaha AR, et al. Current trends in initial management of laryngeal cancer: the declining use of open surgery. Eur Arch Otorhinolaryngol 2009;266:1333-52.

14. Thomas L, Drinnan M, Natesh B, et al. Open conservation partial laryngectomy for laryngeal cancer: a systematic review of english language literature. Cancer Treat Rev 2012;38:203-11.

15. Wen WP, Su ZZ, Zhu XL, et al. Supracricoid partial laryngectomy with cricothyroidopexy: a treatment for anterior vocal commissure laryngeal squamous carcinoma. Head Neck 2013;35:311-5.

16. Strong MS, Jako GJ. Laser surgery in the larynx. Early clinical experience with continuous CO2 laser. Ann Otol Rhinol Laryngol 1972;81:791.

17. Eckel HE, Thumfart W, Jungehülsing $M$, et al. Transoral laser surgery for early glottic carcinoma. Eur Arch 
Otorhinolaryngol 2000;257:221-6.

18. Motta G, Esposito E, Motta S, et al. CO2 laser surgery in the treatment of glottic cancer. Head Neck 2005;27:733.

19. Pradhan SA, Pai P, Neeli S, et al. Transoral laser surgery for early glottic cancers. Arch Otolaryngol Head Neck Surg 2003;129:623-5.

20. Rudert HH, Werner JA. Endoscopic resections of glottic and supraglottic carcinomas with the CO2 laser. Eur Arch Otorhinolaryngol 1995;252:146-8.

21. Spector JG, Sessions DG, Chao KSC, et al. Stage I (T1N0M0) squamous cell carcinoma of the laryngeal glottis: therapeutic results and voice preservation. Head Neck 1999;21:707-17.

22. Laccourreye O, Muscatello L, Laccourreye L, et al. Supracricoid partial laryngectomy with cricohyoidoepiglottopexy for "early" glottic carcinoma classified as T1-T2N0 invading the anterior commissure. Am J Otolaryngol 1997;18:385-90.

23. Sheen TS, Ko JY, Chang YL. Partial vertical laryngectomy in the treatment of early glottic cancer. Ann Otol Rhinol Laryngol 1998;107:593-7.

24. Spector JG, Sessions DG, Chao KSC, et al. Management of stage II (T2N0M0) glottic carcinoma by radiotherapy and conservation surgery. Head Neck 1999;21:116-23.

25. Thomas JV, Olsen KD, Neel HBR, et al. Early glottic carcinoma treated with open laryngeal procedures. Arch Otolaryngol Head Neck Surg 1994;120:264-8.

26. Rödel RMW, Steiner W, Müller RM, et al. Endoscopic laser surgery of early glottic cancer: involvement of the anterior commissure. Head Neck 2009;31:583-92.

27. Steiner W, Ambrosch P, Rödel RMW, et al. Impact of anterior commissure involvement on local control of early glottic carcinoma treated by laser microresection. Laryngoscope 2004;114:1485-91.

28. Zeitels SM, Franco RA, Hillman RE, et al. Voice and treatment outcome from phonosurgical management of early glottic cancer. Ann Otol Rhinol Laryngol Suppl 2002;190:3-20.

29. Agrawal N, Ha PK. Management of early-stage laryngeal cancer. Otolaryngol Clin North Am 2008;41:757-69.

30. Sachse F, Stoll W, Rudack C. Evaluation of treatment results with regard to initial anterior commissure involvement in early glottic carcinoma treated by external partial surgery or transoral laser microresection. Head Neck 2009;31:531-7.

31. Bergler W, Maleck W, Baker-Schreyer A, et al. The mallampati score. Prediction of difficult intubation in otolaryngologic laser surgery by mallampati score.
Anaesthesist 1997;46:437-40.

32. Lee SL, Hosford C, Lee QT, et al. Mallampati class, obesity, and a novel airway trajectory measurement to predict difficult laryngoscopy. Laryngoscope 2015;125:161-6.

33. Yamamoto K, Tsubokawa T, Shibata K, et al. Predicting difficult intubation with indirect laryngoscopy. Anesthesiology 1997;86:316-21.

34. Fiz I, Koelmel JC, Sittel C. Nature and role of surgical margins in transoral laser microsurgery for early and intermediate glottic cancer. Curr Opin Otolaryngol Head Neck Surg 2018;26:78-83.

35. Fiz I, Mazzola F, Fiz F, et al. Impact of close and positive margins in transoral laser microsurgery for Tis-T2 glottic cancer. Front Oncol 2017;7:245.

36. Grant DG, Repanos C, Malpas G, Salassa JR, et al. Transoral laser microsurgery for early laryngeal cancer. Expert Rev Anticancer Ther 2010;10:331-8.

37. Bjordal K, Graeff AD, Fayers PM, et al. A 12 country field study of the EORTC QLQ-C30 (version 3. 0) and the head and neck cancer specific module (EORTC QLQ-H\&N35) in head and neck patients. Eur J Cancer 2000;36:1796-807.

38. Terwee CB, Dekker FW, Wiersinga WM, et al. On assessing responsiveness of health-related quality of life instruments: guidelines for instrument evaluation. Qual Life Res 2003;12:349-62.

39. Luo J, Wu J, Lv K, et al. Analysis of postsurgical healthrelated quality of life and quality of voice of patients with laryngeal carcinoma. Medicine (Baltimore) J 2016;95:e2363.

40. Lundström E, Hammarberg B, Munck-Wikland E. Voice handicap and health-related quality of life in laryngectomees: assessments with the use of VHI and EORTC questionnaires. Folia Phoniatr Logop 2009;61:83-92.

41. Bindewald J, Oeken J, Wollbrueck D, et al. Quality of life correlates after surgery for laryngeal carcinoma. Laryngoscope 2007;117:1770-6.

(English Language Editor: M. Hawkins)

Cite this article as: Luo C, Lv K, Liu Q, Wen Y, Lin M, Wang Z, Zhu X, Jiang A, Wen W, Lei W. Comparison of laser microsurgery and open partial laryngectomy for T1-2 laryngeal cancer treatment. Ann Transl Med 2021;9(6):464. doi: 10.21037/ atm-21-135 